\title{
The Impact of Ischemic Heart Disease on Rheumatoid Arthritis
}

\author{
Rafael Kmiliauskis Santos Gomes, $P h D^{1^{*}}$ and Moacyr Roberto Cuce Nobre, $P h D^{2}$ \\ ${ }^{1}$ Master in Public Health and Phd in Sciences, Center of Medical Specialties of Brusque and Blumenau, Brazil \\ ${ }^{2}$ Master and PhD in Rheumatology, University of São Paulo, Brazil
}

\begin{abstract}
Rheumatoid arthritis (RA) is a systemic inflammatory autoimmune disease of unknown etiology. Current knowledge supports that chronic inflammation represents a crucial risk factor without prior development of atherosclerosis. Thus, the epidemiological findings of patients with RA have demonstrated an expressive increase in cardiovascular morbidity and mortality. In addition, these patients present a higher prevalence of coronary risk factors and, when associated with poor prognostic factors, increase the risk of ischemic heart disease. The use of immunobiological drugs when compared to synthetic ones seems to decrease a chance of acute myocardial infarction (AMI), especially when there is a longer exposure time. The longer the period in remission or low disease activity, the lower the risk. The time interval between onset of RA and AMI depends on the population being considered in the study. The first evidence of the best form of primary prevention begins to emerge, a reality is different from the secondary one. There are still challenges in the construction of cardiovascular risk calculators specifically for RA patients and the correct ways of adapting the existing ones for the general population. There is a lower risk of death during hospitalization for AMI treatment in relation to the general population, however, this scenario becomes unfavorable in the medium and long terms, both for death and for a new coronary ischemic event.
\end{abstract}

\section{Keywords}

Rheumatoid arthritis, Cardiovascular, Acute myocardial infarction, Epidemiology, Mortality

\begin{abstract}
Abbreviations
RA: Rheumatoid Arthritis; AMI: Acute Myocardial Infarction; TCVFR: Traditional Cardiovascular Risk Factors; CVD: Cardiovascular Disease; CPR: Creactive Protein; ExtAr: Extra Articular Disease; SAH: Systemic Arterial Hypertension; DM2: Type 2 Diabetes Mellitus; DLP: Dyslipidemia; MTX: Methotrexate; CV: Cardiovascular Risk; DMARDs: DiseaseModifying Synthetic Drugs; OR: Odds Ratio; SSZ: Sulfasalazine; HCQ: Hydroxychloroquine; Anti TNF: Anti-Tumor Necrosis Factor Antibody; ACS: Acute Coronary Syndrome; RR: Relative Risk; DAS28: Disease Activity Score for 28-Joint Counts; ESR: Erythrocyte Sedimentation Rate; CDAl: Clinical Disease Activity Index; ASA: Acetylsalicylic Acid; ACEI: Angiotensin Converting Enzyme Inhibitor; ARB2: Angiotensin II Receptor Blockers; EULAR: European League Against Rheumatism; SCORE: Systemic Coronary Risk Evaluation
\end{abstract}

\section{Introduction}

Rheumatoid arthritis (RA) is a systemic inflammatory autoimmune disease of unknown etiology. The prevalence of RA is estimated to be $0.5 \%$ to $1 \%$ of the adult world population, with a prevalence of two to three times higher in women [1]. Current knowledge supports that chronic systemic inflammation represents a crucial risk factor in the early development of atherosclerosis, predisposing to ischemic heart disease [2]. Thus, the epidemiological findings of patients with RA have demonstrated an expressive increase in cardiovascular morbidity and mortality [3].

Traditional cardiovascular risk factors (TCVRF) that include increased age, male sex, sedentary lifestyle, smoking, dyslipidemia, systemic arterial hypertension, diabetes mellitus, and a positive family history are not enough to explain the excess of heart disease [4]. A large multicenter international study concluded that the risk attributable to RA combined with TCVRF was $70 \%$ for cardiovascular disease (CVD). When analyzed separately, the risk remained the same for RA and decreased to $49 \%$ for cardiovascular risk factors [5].

The data indicate that autoimmune rheumatologic diseases present a greater number of cases of ischemic heart

*Corresponding author: Rafael Gomes, Master in Public Health and PhD in Sciences, Center of Medical Specialties of Brusque and Blumenau, Dois de Setembro 1234, 3rd floor, room 307, Itoupava Norte, 89052-003, Blumenau, Santa Catarina, Brazil

Accepted: February 19, 2019

Published online: February 19, 2019

Citation: Gomes RKS, Nobre MRC (2019) The Impact of Ischemic Heart Disease on Rheumatoid Arthritis. Ann Heart 4(1):79-85 
diseases. A retrospective cohort study with a database from Australia conducted between 2001 and 2007 with 79,390 individuals with a first episode of acute myocardial infarction (AMI), where $1.8 \%$ had autoimmune rheumatologic diseases, and among them, RA was the more frequent with $52.2 \%$ of the cases [6].

The UK cohort study (CALIBER) from 1997 to 2010 was constructed through a linkage between basic care, hospital and mortality information systems involving a sample with RA $(12,120$ patients) compared to the non-RA population (121,191 individuals) with an average follow-up of 4 years to evaluate all manifestations of cardiovascular disease, only AMI, heart failure and sudden death are at higher risk [7].

A meta-analysis and a systemic review published in 2008 involving RA patients reported a standardized mortality rate of cardiovascular diseases $50 \%$ and a higher incidence of fatal AMI when compared to the general population, respectively $[8,9]$.

This article aims to update knowledge about prevalence and incidence; traditional cardiovascular risk factors, biological factors directly related to the disease, poor prognostic factors, persistence of inflammatory disease activity, drug treatment and disease duration, AMI treatment, primary and secondary prevention, calculator for risk estimation, cardiovascular mortality and their correlations to determine the increase in ischemic heart risk in RA according to the schematic model proposed in Figure 1. A review of the literature was performed in the Pubmed database, using the following keywords: rheumatoid arthritis, cardiovascular, acute myocardial infarction, epidemiology and mortality.

\section{Discussion}

\section{Prevalence and incidence}

The prevalence of AMI worldwide among RA patients obtained from data of a self-reported survey conducted between January 2005 and October 2006 with 4363 patients in 15 countries was $3.2 \%$ [10]. Previous studies have shown that European countries have a prevalence of between $2 \%$ (United Kingdom) and 7\% (Germany and the Netherlands) [10-12] African countries from 1\% (Morocco) to 3\% (Egypt) [10], Asian countries (South Korea) to 5\% (Japan and Russia) $[10,13,14]$, North America (United States) between 3.7\% and $5 \%[10,15,16]$ and finally Latin America between $2 \%$ (Argentina and Venezuela) and 7\% (Uruguay) [10,17].

The high incidence of AMI among the English patients, being observed in 5.52 cases per 1000 thousand people/year when compared to the general population of 2.91 cases 7 . The incidence and relative risk for the occurrence of RA patients presenting with AMI is higher than the general population $[7,18]$. Recent meta-analysis indicates that the relative risk is of the order of $52 \%$ even after adjusted analysis for sex, age and CVRF [19].

However, data from the Japanese population showed a

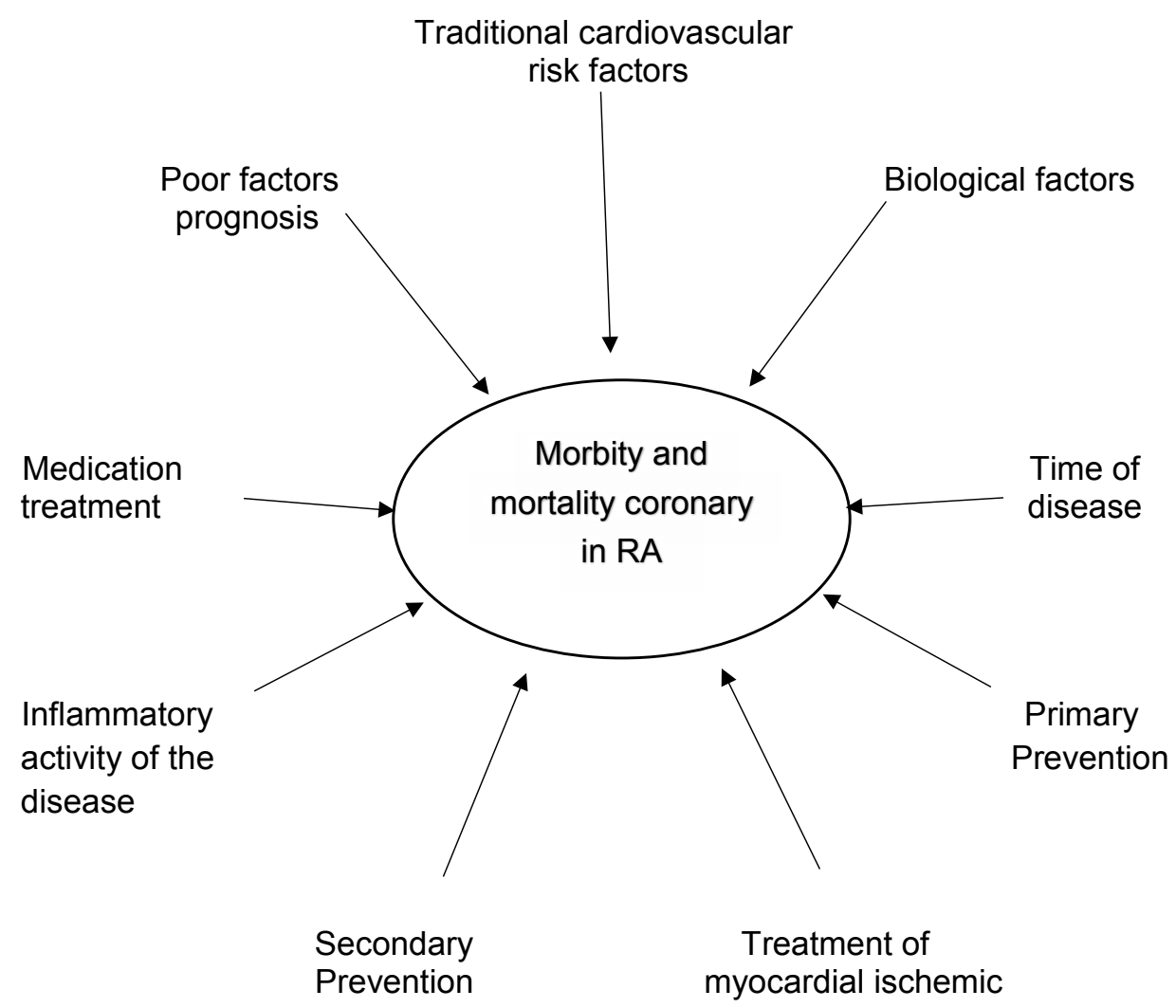

Figure 1: Schematic model of the relevant factors in coronary ischemic morbidity and mortality among patients with rheumatoid arthritis.

RA: Rheumatoid Arthritis. 
lower incidence, 2.2 cases per 1000 thousand people/year, denoting a different scenario from the western world [20].

\section{Specific biological factors related to rheumatoid arthritis}

Increased C-reactive protein (CRP), proinflammatory cytokines, tumor necrosis factor alpha and interleukin 6 are important factors in atherosclerosis in RA and are considered independent markers of the future coronary event. In addition, the unstable atherosclerotic plaque and the rheumatoid synovial membrane share similar scenarios of deregulation of local and systemic immune activation due to the accumulation of monocytes, macrophages and T cells and an extracellular increase of metalloprotein degradation, changes that contribute to accelerated atherosclerosis. The Increased carotid arteries intima-media thickness and calcium deposition in the coronary arteries when compared to individuals without RA, lead to increased plaque instability in RA patients. Additionally, a pro-atherogenic profile is established: lipid abnormalities, oxidative stress, depletion of endothelial progenitor cells, increased arterial stiffness, increased insulin resistance, endothelial dysfunction, hypercoagulability, elevated homocysteine levels, and atherogenic T-cell overexpression [21]. A post-death analysis indicated a higher density of inflammatory cells at the border of the area of myocardial necrosis after an episode of AMI among RA patients than in the control group [22].

\section{Presence of poor prognostic factors related to rheumatoid arthritis}

The greatest number of cardiac ischemic events in RA patients is not entirely explained by the presence of TCVRF. There is a potentiality relationship between these factors and the disease severity markers both isolated, but especially when both are present at the onset of the disease [15].

The QUEST RA study correlated that the patient with extra-articular disease (ExtAr) presented a greater chance (116\%) of AMI when compared to the patient without ExtAr [10]. Similarly, Solomon DH, et al. through a recording study in the United States called CORRONA, positively related that the greater the presence of risk factors associated with poor prognosis, both determine a progressive increase in the rate of AMI [15]. These findings were again confirmed with a special focus on the most common manifestation ExtAr, the rheumatoid nodule. Patients with a subcutaneous nodule present a $65 \%$ higher incidence of $\mathrm{AMI}$ and $68 \%$ for cardiovascular death than those who did not present it [23].

\section{Presence of traditional cardiovascular risk fac- tors}

A Swedish study reveals that at the time of diagnosis, $53.2 \%$ of patients with RA have 1 or more morbidities, in addition to developing a substantial increase in new morbidities in the first 5 years of disease [12].

The national epidemiological data point to a higher prevalence of TCVRF among RA patients. Data from a cohort study conducted in Blumenau, Santa Catarina, in the year
2015 [24] when comparing the general Brazilian population obtained through the population telephone survey on the frequency of risk factors for chronic diseases (VIGITEL) of 2016 [25] show the following differences of prevalence respectively: Systemic arterial hypertension (SAH) $44.8 \%$ versus $25.7 \%$, type 2 diabetes mellitus (DM2) 13.2\% versus $8.9 \%$, dyslipidemia (DLP) $27.4 \%$ versus $20.3 \%$, obesity $21.2 \%$ versus $18.9 \%$; previous smoking $41.1 \%$ versus $23.4 \%$, current smoking $11.1 \%$ versus $10.2 \%$ and sedentary lifestyle $66.9 \%$ versus $62.4 \%$. The influence of each of the TCVRF was analyzed in a multicenter study that proved that dyslipidemia and smoking were associated with AMI [10]. However recent systemic review article and meta-analysis establishes that the risk of AMI increases among patients with SAH and DM2 [26], this last risk factor coincided with the Blumenau study [24].

\section{Medications for rheumatoid arthritis and time of exposure}

The Interest in the effect of antirheumatic drugs on the inflammatory response and its relationship to cardiovascular risk arises from observations of the association between the use of methotrexate (MTX) and the reduction of cardiovascular risk (CV) in RA [27]. The cardioprotective action is uncertain but may occur by reducing the systemic inflammation of CRP levels, interleukin 6 and tumor necrosis factor alpha, and by the direct effect of some cellular mechanisms leading to atherosclerosis. Evidence points out to this same protective effect when MTX is used in low doses in the non-RA population. With earlier treatment of MTX disease, there may be a long-term change in cardiovascular risk [28]. In addition to exposure to MTX alone, combined treatment of disease-modifying synthetic drugs (DMARDs) is associated with a significant reduction in cardiovascular risk with odds ratio (OR): 0.16 with $\mathrm{MTX}$ alone, OR: 0.20 with MTX and sulfasalazine (SSZ); and OR: 0.20 with MTX, SSZ and hydroxychloroquine (HCQ) [29]. Data from a meta-analysis conducted with the objective of evaluating the cardiovascular effect in RA including methotrexate as representative of DMARDs and anti-tumor necrosis factor antibody (anti-TNF), on the part of immunobiologicals, it revealed a relative risk reduction for $\mathrm{AMI}$ of $19 \%$ for the first and $41 \%$ for the second [30].

There are studies indicating that the anti-TNF drug reduces the risk of cardiovascular event and this may be related to the drug's time use [31-33]. The survival rate of patients on the active use of anti-TNF free of acute coronary syndrome (ACS), when compared to patients who have never used biological, demonstrate a growing difference over time. Patients who used anti-TNF during the first 2, 4, 6 and 8 years of disease had a free ACS rate of $1.75,2.54,1.73$ and 1.71 versus $2.67,2.54,2.14$ and 5.31 of those who never used immunobiological. Another relevant factor besides of the drug's action was that the exposure time may also influence coronary ischemic outcome. When the user is less than or equal to 16 months, the relative risk (RR) for the incidence of ACS is 0.42 , while the exposure greater than 16 months determines an RR of 0.18 [27]. On the other hand, some drugs used for joint pain relief, such as glucocorticoid, increases the relative risk 
of $\mathrm{AMI}$ and non-steroidal anti-inflammatory drugs by $41 \%$ in $13 \%[30]$.

The joint analysis of three large health databases in the United States concluded that there was no difference in the risk of AMI between tocilizumab versus anti-TNF [3]. However, the effect of immunobiologicals may vary according to the age group of the patient. The comparison for AMI at the start of immunobiological treatment among patients older than 65 years may be associated with a $30 \%$ higher risk for the anti-TNF compared to the co-stimulation blocker. When analyzed jointly with $\mathrm{AMI}$, percutaneous coronary intervention and myocardial revascularization surgery, the anti-interleukin 6 drug presented a $36 \%$ risk reduction when compared to the co-stimulation blocker [34].

\section{Period of permanence in remission or inflamma- tory activity of the disease}

The high inflammatory activity of the disease and the time of exposure represent important determinants for increased cardiovascular risk. A cohort study conducted in Sweden, including patients who presented a diagnosis of RA between 1996 and 2011, was carried out to evaluate the effect of disease activity, measured by disease activity score for 28-joint counts (DAS 28), at risk of the first episode of SCA. During the first year of illness, high disease activity was associated with an increased risk of ACS. When disease activity was assessed in the total follow-up period, an association between high disease activity and ACS remained [35]. The cardiovascular event at early RA (less than 12 months of symptoms until diagnosis) can be explained by the influence of activity according to data from a cohort study of patients followed up for 5 years. After adjusted analysis, the impact of high activity and the exposure time established by DAS 28 , especially influenced by the erythrocyte sedimentation rate (ESR) component at the onset of the disease, are independent factors for the increased risk of ischemic cardiac event [36].

Similarly, a study in the Netherlands presented a result that confirms that the mean time of exposure to high disease activity represented by DAS 28 is an independent variable with a significant effect on the risk of CVD. After correction for confounding factors, for each point of increase of DAS 28 , the risk increased by 0.281 [37]. A cohort of patients with RA from the United States followed on average for 2 years and 7 months, found a significant CV risk reduction for each 10-point reduction in the Clinical Disease Activity Index (CDAI). Compared to high levels of disease activity, the moderate disease had a $35 \%$ reduction, a low disease activity $58 \%$ and a disease remission of $60 \%$, which shows a decreasing and proportional reduction at all levels of activity of disease [38].

\section{Time of disease}

The risk of AMI increases rapidly from the diagnosis of RA suggesting additional disease-related mechanism for accelerated development of atherosclerosis. A Swedish follow-up study of patients with initial RA between 1995 and 2006 including 7469 patients indicated that the increased relative risk for $A M I$ already occurs between the first and fourth year of disease with an average time of 4.8 years [39], thus establishing that in around $75 \%$ of cases of AMI occurred in the first 5 years of illness. The influence of diagnostic time for the first CV event was also documented through the 11year mean follow-up analysis in Japan. After multivariate regression analysis for confounding factors, disease time greater than 10 years was an independent risk factor [40]. For the national reality, the Blumenau cohort study also demonstrates the association of disease time greater than 10 years and AMI [24].

However, these findings were comforted by the Dutch cohort. The evidence for this country indicates that the duration of the disease does not independently affect CV risk when the cutoff point chosen was the first 10 years of disease [37].

\section{Primary and secondary prevention}

An English study was conducted among RA patients aged 60 years or older to assess the effect of acetylsalicylic acid (ASA) on primary prevention of AMI. The results do not establish any protective effect [41]. Another drug targeted for primary prevention was statins. A systematic review carried out for this purpose pointed out that current evidence seems to establish a CV risk reduction in primary prevention in high doses, but without effect for secondary prevention either with high or low doses and that in addition the discontinuity of the medication increased the risk for AMI [42]. The most recent evidence indicates similarly what it happens to the general population for the angiotensin-converting enzyme inhibitor (ACEI), and angiotensin II receptor blockers (ARB2) used in hypertensive patients with RA reduce $A M I$ by the order of $30 \%$ with ACE inhibitors, $36 \%$ with ARB2 and $37 \%$ when associated. This protective effect was significantly higher when used for a long time [43].

From the moment that RA patients had AMI, those present a higher prevalence of worse cardiac remodeling parameters such as: Ventricular hypertrophy, diastolic dysfunction, lower ejection fraction, higher incidence of tricuspid and pulmonary valve regurgitation than patients breathless. To minimize these deleterious effects, inflammatory control of arthritis should occur as rapidly as possible [44]. Another significant difference that may contribute to the worst outcome after AMI was documented in Australia after data analysis from 1995 to 2005 . Patients with AR received fewer beta-blocker and statins medications for secondary prevention than subjects who did not have RA during hospital admission. This situation remained similar in the subgroup analysis among the patients who had undergone the first $\mathrm{AMI}$, since during the second AMI admission, less ASA, beta-blocker and statin were used [45].

\section{Cardiovascular risk calculators}

The trans-Atlantic study consortium for cardiovascular risk in RA performed a combination of 13 cohorts in 10 different countries with the intention of constructing a specific cardiovascular risk calculator for RA, but the challenge remained because the performance of the constructed models was similar to the calculators used in the general population [46]. This initiative arose from the previous observation that the 
calculators directed to RA were not superior to the general calculators [47]. An example of this occurred when comparing the performance of two established CV risk calculators for the general population, a Framingham study, and American College of Cardiology/American Heart Association, among patients with RA, with a disagreement of approximately $10 \%$ of the cases [48]. Even with the European League Against Rheumatism (EULAR 2015-2016) recommendation that guides the multiplication of 1.5 to the obtained result, there was a variability between 6 different calculators in the studied Mexican population, thus could not be used indistinctly in patients with RA [49]. In the national scenario, a study was carried out to analyze the predictor of fatal CVD for women with RA versus control group using Systemic Coronary Risk Evaluation (SCORE) original and modified, as suggested by EULAR, for using disease characteristics. The outcome of the former was similar for both groups, but the second presented a greater risk, which reinforces the importance of considering disease-related factors [50].

\section{Treatment of AMI in rheumatoid arthritis}

The treatment of AMI performed in the period between 1995 and 2005 in the RA population occurred differently from the control group. Rheumatologic patients were less likely to receive acute reperfusion, thrombolysis or percutaneous coronary intervention with or without stent implantation within 12 hours after the onset of symptoms, even after an adjusted analysis for type, prior presence and clinical presentation of AMI. There was no significant difference for treatment with angioplasty or myocardial revascularization [45]. In the same period, another study conducted in the United States was designed to establish the effect of RA on the choice of AMI treatment. Patients with RA were more likely to undergo thrombolysis and percutaneous coronary intervention [51]. Later, between 2010 and 2014, in Japan, another analysis was done to assess how much AR morbidity affects the likelihood of undergoing reperfusion therapy of coronary artery disease. The findings showed that there was no difference between the rheumatoid patients and the control group [52]. All of these evidences lead to speculation that the type of AMI treatment may vary according to the time and country in which it was performed.

\section{Mortality rate and the possible influence of AMI treatment}

A cross-sectional analysis from 2003 to 2005 conducted in the United States, including 1,112,676 AMI patients, revealed that RA patients had a $24 \%$ lower in-hospital mortality rate compared to non-RA patients, and this value increased to $34 \%$ after adjusted analysis for variables of confusion. These results were significantly associated with those who underwent drug treatment and percutaneous intervention, but not for thrombolysis and revascularization surgery. When this same study specifically examines patients undergoing revascularization, RA patients have a $49 \%$ lower chance of in-hospital death, with $61 \%$ less chance for percutaneous intervention and $31 \%$ for second revascularization surgery [51].
A study conducted in Victoria, Australia, between 2001 and 2003, involving 29,924 patients, 359 (1.2\%) with RA presented the first acute cardiovascular event, defined by acute myocardial infarction, the 30-day cardiovascular mortality rate was $21.3 \%$, higher than the general population, which was $10.5 \%$, with a chance ratio of 1.9 . In addition, the all-cause mortality rate after 30 days of AMI was $22.7 \%$, and the odds ratio was 1.8 after an adjusted analysis for sex, age, cardiovascular risk factors and type of cardiac treatment performed [53].

This scenario did not show significant changes even after the extension of the observation period from 2001 to 2007 and number of patients analyzed (79.390). The shortterm results show that, for all-cause death as well as for cardiovascular causes, RA patients present a $50 \%$ greater chance than the general population. Both the medium-term, first 12-month and long-term outcomes, longer than 5 years, remain unfavorable to RA patients. The result in the medium term, mortality increases to $100 \%$ for all causes and $85 \%$ for CV cause [6].

Even receiving similar reperfusion intervention and cardioprotective medications for secondary prevention, patients with RA have worse long-term outcomes. The mortality rate and the cumulative incidence difference of ischemic recurrence showed a significant increase from the $5^{\text {th }}$ year of myocardial infarction among patients with RA [54], especially for those undergoing percutaneous coronary intervention [55] but not for revascularization surgery [56].

\section{Conclusion}

Among all presentations of cardiovascular diseases, AMI is the most common manifestation in RA. These patients have a higher incidence due to a more accelerated atherosclerotic process, a more unstable atherosclerosis plaque, and the higher prevalence of TCVRF, especially when associated with poor prognostic factors that increase risk.

Prolonged anti-TNF exposure when compared to DMARDs seems to reduce the risk of $\mathrm{AMI}$, but for the remaining non-anti-TNF immunobiologicals, more robust data are still needed. Another important point is related to disease activity and the time of exposure to it. The longer the period in remission or low disease activity, the lower the risk. The time interval between onset of RA and AMI depends on the population being considered in the study. The first evidence for the best form of primary prevention begins to emerge, a different reality from the secondary one. The construction of specific cardiovascular risk calculators for RA patients and the correct ways to adapt existing ones for the general population remains a challenge.

Patients with RA have a lower risk of death during hospitalization for AMI, but this scenario becomes unfavorable in the medium and long term, since there is a higher risk of death and a new coronary ischemic event.

\section{Declarations}

Not applicable. 


\section{Ethics Approval and Consent to Participate}

Not applicable.

\section{Consent for Publication}

Not applicable.

\section{Availability of Data and Material}

Literature review.

\section{Competing Interests}

Not applicable.

\section{Funding}

Not applicable.

\section{Authors' Contributions}

RKSG and MRCN also contributed in all stages of the article: Elaboration, literature review, evidence analysis, article writing and final approval for submission to the journal.

\section{Acknowledgements}

Not applicable.

\section{References}

1. Bertolo MB, Brenol CV, Schainberg CG, et al. (2007) Atualização do consenso brasileiro no diagnóstico e tratamento de artrite reumatoide. Rev Bras Reumatol 47: 151-159.

2. Ozbalkan Z, Efe C, Cesur M, et al. (2010) An update on the relationships between rheumatoid arthritis and atherosclerosis. Atherosclerosis 212: 377-382.

3. Kim SC, Solomon DH, Rogers JR, et al. (2017) Cardiovascular safety of tocilizumab versus tumor necrosis factor inhibitors in patients with rheumatoid arthritis: A Multi-Database cohort study. Arthritis Rheumatol 69: 1154-1164.

4. Symmons DP, Gabriel SE (2011) Epidemiology of CVD in rheumatic disease, with a focus on RA and SLE. Nat Rev Rheumatol 7: 399408.

5. Crowson CS, Rollefstad S, Ikdahl E, et al. (2018) Impact of risk factors associated with cardiovascular outcomes in patients with rheumatoid arthritis. Ann Rheum Dis 77: 48-54.

6. Van Doornum, Bohensky M, Tacey MA, et al. (2015) Increased 30-day and 1-year mortality rates and lower coronary revascularisation rates following acute myocardial infarction in patients with autoimmune rheumatic disease. Arthritis Res Ther 17: 38.

7. Pujades-Rodriguez M, Duyx B, Thomas SL, et al. (2016) Rheumatoid arthritis and incidence of twelve initial presentations of cardiovascular disease: A population record-linkage cohort study in England. PLoS One 11: e0151245.

8. Aviña-Zubieta JA, Choi HK, Sadatsafavi M, et al. (2008) Risk of cardiovascular mortality in patients with rheumatoid arthritis: A meta-analysis of observational studies. Arthritis Rheum 59: 1690-1697.

9. Lévy L, Fautrel B, Barnetche T, et al. (2008) Incidence and risk of fatal myocardial infarction and stroke events in rheumatoid arthritis patients. A systematic review of the literature. Clin Exp Rheumatol 26: 673-679.
10. Naranjo A, Sokka T, Descalzo MA, et al. (2008) Cardiovascular disease in patients with rheumatoid arthritis: Results from the QUEST-RA study. Arthritis Res Ther 10: 30.

11. Balsa A, Lojo-Oliveira L, Alperi-López M, et al. (2017) Prevalence of Comorbidities in Rheumatoid Arthritis and Evaluation of Their Monitoring in Clinical Practice: The Spanish Cohort of the COMORA Study. Reumatol Clin 258: 30134-30141.

12. Innala L, Sjöberg C, Möller B, et al. (2016) Co-morbidity in patients with early rheumatoid arthritis - inflammation matters. Arthritis Res Ther 18: 33

13. Jeong H, Baek SY, Kim SW, et al. (2017) Comorbidities of rheumatoid arthritis: Results from the Korean National Health and Nutrition Examination Survey. PLoS One 12: 0176260.

14. Panafidina TA, Kondratyeva LV, Gerasimova EV, et al. (2013) Characteristics and cardiovascular comorbidities in patients with rheumatoid arthritis in a local patient cohort in Russia. Ann Rheum Dis 72: 865-866.

15. Solomon DH, Kremer J, Curtis JR, et al. (2010) Explaining the cardiovascular risk associated with rheumatoid arthritis: Traditional risk factors versus markers of rheumatoid arthritis severity. Ann Rheum Dis 69: 1920-1925.

16. Dougados M, Soubrier M, Antunez A, et al. (2014) Prevalence of comorbidities in rheumatoid arthritis and evaluation of their monitoring: Results of an international, cross-sectional study (COMORA). Ann Rheum Dis 73: 62-68.

17. Sarmiento-Monroy JC, Amaya-Amaya J, Espinosa-Serna JS, et al. (2012) Cardiovascular disease in rheumatoid arthritis: A systematic literature review in latin america. Arthritis 371909: 1-17.

18. Chung WS, Lin CL, Peng CL, et al. (2013) Rheumatoid arthritis and risk of acute myocardial infarction-a nationwide retrospective cohort study. Int J Cardiol 168: 4750-4754.

19. Schieir O, Tosevski C, Glazier RH, et al. (2017) Incident myocardial infarction associated with major types of arthritis in the general population: A systematic review and meta-analysis. Ann Rheum Dis 76: $1396-1404$.

20. Sugimoto N, Nakajima A, Inoue E, et al. (2017) Incidence of comprehensive hospitalization due to infection, cardiovascular disease, fractures, and malignancies in patients with rheumatoid arthritis. Rheumatol Int 37: 1871-1878.

21. Ku IA, Imboden JB, Hsue PY, et al. (2009) Rheumatoid arthritis: Model of systemic inflammation driving atherosclerosis. Circ $\mathrm{J}$ 73: 977-985.

22. Van den Oever IA, van Sijl AM, Baylan U, et al. (2016) Comparing inflammatory cell density in the myocardium and coronary arteries in rheumatoid arthritis patients versus controls with myocardial infarction: A post-mortem case-control study. Int J Cardiol 209: 74-76.

23. Kaushik P, Solomon DH, Greenberg JD, et al. (2015) Subcutaneous nodules are associated with cardiovascular events in patients with rheumatoid arthritis: Results from a large US registry. Clin Rheumatol 34: 1697-1704.

24. Gomes RKS, Albers AC, Salussoglia AIP, et al. (2017) Prevalence of ischemic heart disease and associated factors in patients with rheumatoid arthritis in Southern Brazil. Rev Bras Reumatol 57: 412-418.

25. Ministério da Saúde (2016) Hábitos dos brasileiros impactam no crescimento da obesidade e aumenta prevalência de diabetes e hipertensão. Vigitel Brasil. 
26. Baghdadi LR, Woodman RJ, Shanahan EM, et al. (2015) The impact of traditional cardiovascular risk factors on cardiovascular outcomes in patients with rheumatoid arthritis: A systematic review and meta-analysis. PLoS One 10: e0117952.

27. Bili A, Tang X, Pranesh S, et al. (2014) Tumor necrosis factor a inhibitor use and decreased risk for incident coronary events in rheumatoid arthritis. Arthritis Care Res 66: 355-363.

28. Marks JL, Edwards CJ (2012) Protective effect of methotrexate in patients with rheumatoid arthritis and cardiovascular comorbidity. Ther Adv Musculoskelet Dis 4: 149-157.

29. Van Halm VP, Nurmohamed MT, Twisk JW, et al. (2006) Diseasemodifying antirheumatic drugs are associated with a reduced risk for cardiovascular disease in patients with rheumatoid arthritis: A case control study. Arthritis Res Ther 8: 151.

30. Roubille C, Richer V, Starnino T, et al. (2015) The effects of tumour necrosis factor inhibitors, methotrexate, non-steroidal anti-inflammatory drugs and corticosteroids on cardiovascular events in rheumatoid arthritis, psoriasis and psoriatic arthritis: A systematic review and meta-analysis. Ann Rheum Dis 74: 480-489.

31. Greenberg JD, Kremer JM, Curtis JR, et al. (2011) Tumour necrosis factor antagonist use and associated risk reduction of cardiovascular events among patients with rheumatoid arthritis. Ann Rheum Dis 70: 576-582.

32. Sattin M, Towheed T (2016) The Effect of TNF $\alpha$-Inhibitors on Cardiovascular Events in Patients with Rheumatoid Arthritis: An Updated Systematic Review of the Literature. Curr Rheumatol Rev 12: 208-222.

33. Nurmohamed M, Bao $\mathrm{Y}$, Signorovitch J, et al. (2015) Longer durations of antitumour necrosis factor treatment are associated with reduced risk of cardiovascular events in patients with rheumatoid arthritis. RMD Open 1: 1-9.

34. Zhang J, Xie F, Yun H, et al. (2016) Comparative effects of biologics on cardiovascular risk among older patients with rheumatoid arthritis. Ann Rheum Dis 75: 1813-1818.

35. Mantel $\ddot{A}$, Holmqvist M, Nyberg F, et al. (2015) Risk factors for the rapid risk increase of acute coronary events in patients with new-onset rheumatoid arthritis: A nested case-control study. Arthritis Rheumatol 67: 2845-2854.

36. Innala L, Möller B, Ljung L, et al. (2011) Cardiovascular events in early RA are a result of inflammatory burden and traditional risk factors: A five year prospective study. Arthritis Res Ther 13: 131.

37. Arts EE, Fransen J, den Broeder AA, et al. (2015) The effect of disease duration and disease activity on the risk of cardiovascular disease in rheumatoid arthritis patients. Ann Rheum Dis 74: 998-1003.

38. Solomon DH, Reed GW, Kremer JM, et al. (2015) Disease activity in rheumatoid arthritis and the risk of cardiovascular events. Arthritis Rheumatol 67: 1449-1455.

39. Holmqvist ME, Wedrén S, Jacobsson LT, et al. (2010) Rapid increase in myocardial infarction risk following diagnosis of rheumatoid arthritis amongst patients diagnosed between 1995 and 2006. J Intern Med 268: 578-585.

40. Masuda H, Miyazaki T, Shimada K, et al. (2014) Disease duration and severity impacts on long-term cardiovascular events in Japanese patients with rheumatoid arthritis. J Cardiol 64: 366-370.

41. Durán J, Peloquin C, Zhang Y, et al. (2017) Primary Prevention of Myocardial Infarction in Rheumatoid Arthritis Using Aspirin: A Case-crossover Study and a Propensity Score-matched Cohort Study. J Rheumatol 44: 418-424.
42. Danninger K, Hoppe UC, Pieringer H (2014) Do statins reduce the cardiovascular risk in patients with rheumatoid arthritis? Int J Rheum Dis 17: 606-611.

43. Lin TT, Wu CK, Liao MT, et al. (2017) Primary prevention of myocardial infarction with angiotensin-converting enzyme inhibitors and angiotensin receptor blockers in hypertensive patients with rheumatoid arthritis-A nationwide cohort study. PLoS One 12: E0188720.

44. Pan L, Wang $T$ (2017) Features of cardiac remodeling in patients with acute coronary syndrome complicated with rheumatoid arthritis. Sci Rep 7: 10268.

45. Van Doornum S, Brand C, Sundararajan V, et al. (2010) Rheumatoid arthritis patients receive less frequent acute reperfusion and secondary prevention therapy after myocardial infarction compared with the general population. Arthritis Res Ther 12: 183.

46. Crowson CS, Rollefstad S, Kitas GD, et al. (2017) Challenges of developing a cardiovascular risk calculator for patients with rheumatoid arthritis. PLoS One 12: E0174656.

47. Crowson CS, Gabriel SE, Semb AG, et al. (2017) Rheumatoid arthritis-specific cardiovascular risk scores are not superior to general risk scores: A validation analysis of patients from seven countries. Rheumatology (Oxford) 56: 1102-1110.

48. Jafri K, Ogdie A, Qasim A, et al. (2018) Discordance of the framingham cardiovascular risk score and the 2013 american college of cardiology/american heart association risk score in systemic lupus erythematosus and rheumatoid arthritis. Clin Rheumatol 37: 467-474.

49. Galarza-Delgado DA, Azpiri-Lopez JR, Colunga-Pedraza IJ, et al. (2017) Assessment of six cardiovascular risk calculators in mexican mestizo patients with rheumatoid arthritis according to the EULAR 2015/2016 recommendations for cardiovascular risk management. Clin Rheumatol 36: 1387-1393.

50. de Campos OA, Nazário NO, de Magalhães Souza Fialho SC, et al. (2016) Assessment of cardiovascular risk in patients with rheumatoid arthritis using the SCORE risk index. Rev Bras Reumatol 56: 138-144.

51. Francis ML, Varghese JJ, Mathew JM, et al. (2010) Outcomes in patients with rheumatoid arthritis and myocardial infarction. Am J Med 123: 922-928.

52. Isogai T, Matsui H, Tanaka H, et al. (2017) Treatments and inhospital mortality in acute myocardial infarction patients with rheumatoid arthritis: A nationwide retrospective cohort study in Japan. Clin Rheumatol 36: 995-1004.

53. Van Doornum S, Brand C, King B, et al. (2006) Increased case fatality rates following a first acute cardiovascular event in patients with rheumatoid arthritis. Arthritis Rheum 54: 2061-2068.

54. McCoy SS, Crowson CS, Maradit-Kremers H, et al. (2013) Longterm outcomes and treatment after myocardial infarction in patients with rheumatoid arthritis. J Rheumatol 40: 605-610.

55. Lai CH, Lai WW, Chiou MJ, et al. (2016) Outcomes of percutaneous coronary intervention in patients with rheumatoid arthritis and systemic lupus erythematosus: An 11-year nationwide cohort study. Ann Rheum Dis 75: 1350-1356.

56. Lai CH, Lai WW, Chiou MJ, et al. (2015) Outcomes of coronary artery bypass grafting in patients with inflammatory rheumatic diseases: An 11-year nationwide cohort study. J Thorac Cardiovasc Surg 149: 859-866. 